\title{
Performance evaluation of wireless local area network with congested fading channels
}

\author{
Chanintorn Jittawiriyanukoon ${ }^{1}$, Vilasinee Srisarkun² \\ ${ }^{1}$ Graduate School of Business and Advanced Technology Management, Assumption University, Bangkok, Thailand \\ ${ }^{2}$ Martin de Tours School of Management and Economics, Assumption University, Bangkok, Thailand
}

\begin{tabular}{l} 
Article Info \\
\hline Article history: \\
Received Jul 19, 2020 \\
Revised May 24, 2021 \\
Accepted Jul 1, 2021 \\
\hline
\end{tabular}

Keywords:

Congested network

Fading channels

Multipath communication

Packet error rate

Performance evaluation

Signal-to-noise ratio

WLAN

\begin{abstract}
The IEEE 802.11ay wireless communication standard consents gadgets to link in the spectrum of millimeter wave (mm-Wave) 60 Giga Hertz band through 100 Gbps bandwidth. The development of promising high bandwidth in communication networks is a must as QoS, throughput and error rates of bandwidth-intensive applications like merged reality (MR), artificial intelligence (AI) related apps or wireless communication boggling exceed the extent of the chronic 802.11 standard established in 2012. Thus, the IEEE 802.11ay task group committee has newly amended recent physical (PHY) and medium access control (MAC) blueprints to guarantee a technical achievement especially in link delay on multipath fading channels (MPFC). However, due to the congestion of super bandwidth intensive apps such as IoT and big data, we propose to diversify a propagation delay to practical extension. This article then focuses on a real-world situation and how the IEEE 802.11ay design is affected by the performance of mm-Wave propagation. In specific, we randomize the unstable MPFC link capacity by taking the divergence of congested network parameters into account. The efficiency of congested MPFC-based wireless network is simulated and confirmed by advancements described in the standard.
\end{abstract}

This is an open access article under the CC BY-SA license.

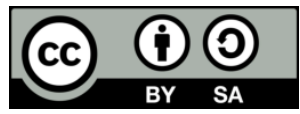

\section{Corresponding Author:}

Chanintorn Jittawiriyanukoon

Graduate School of Business and Advanced Technology Management, Assumption University

Bangkok, Thailand

Email: pct2526@yahoo.com

\section{INTRODUCTION}

Currently, the increment of wireless communication gadgets reflects the fluctuation of network traffic and drives huge demands on the millimeter wave ( $\mathrm{mm}$-Wave) band to attain healthier performance and mitigate congestion problems of wireless mechanisms [1]. Consequently, there are constant developments in appreciating the $5 \mathrm{G}$ mobile networks which are anticipated to support thousand times faster in fundamentally transferring than the traditional 4G [2]. However, wireless networks in this band are particularly provoking compared to microwave band based wireless operation. IEEE 802.11ay is a multi Gbps-wireless network convention at $60 \mathrm{GHz}$ band (that is $57-71 \mathrm{GHz}$ spectrum). Not to mention it is supporting idealistic maximum 300 Gbps transfer rate, advancing through innovative physical (PHY) and medium access control (MAC) layers with multiple-input multiple-output (MIMO) technique, high order-modulation specification, and multipath fading channels (MPFC). The new technology also supports bandwidth intensive applications such as real-time online gaming, $8 \mathrm{~K}$ video streaming, big data analytics, and merged reality. It is regarded that availing the undergone mm-Wave spectrum band (that is $30-300 \mathrm{GHz}$ ) is favorable strategy to comfort intensive bandwidth requests [3]. IEEE 802.11 ad standard also supports $60 \mathrm{GHz}$ band spectrum as defined in [4], which introduces multiGbps Wi-Fi bandwidth to the next generation wireless networks. Its PHY utilizes orthogonal frequency 
division multiplexing (OFDM) scheme [5] and single carrier (SC) [6] mechanism. Although it provides ultrahigh throughput for applications ranging from instant file synchronization to multimedia stream but it is problematic for front-back-hauling wireless applications. The aims of new age wireless networks include communication links with super high-speed, ubiquitous, limited delay, and high capacity. The mm-Wave band spectrum satisfies these necessities and covers for applications the classical wireless technologies (4G/LTE) cannot support. Studying mm-Wave spectrum under IEEE 802.11ay guideline in a PHY level requires distinct simulator to define abstraction models for the impacts of those technologies. Simulators employed by the network analyst do not embrace multi-users MIMO (MU-MIMO) communications. Besides, it involves SNRto-PER environment to testify single-user MIMO (SU-MIMO) case [7].

As appeared in this work regarding IEEE 802.11ay, mm-Wave $60 \mathrm{GHz}$ signal is enhanced directional multi-gigabit (EDMG) to benefit beam-forming signal to noise ratio (SNR) gain and improve path as well as propagation losses for $22 \mathrm{~dB}$ signal [8]. It also escalates the maximum bandwidth to $100 \mathrm{Gbps}$ through data stream on MPFC. The $14 \mathrm{GHz}$ spectrum band is allotted to multiple channels (i.e., 2.16, 4.32, 6.48, and $8.64 \mathrm{GHz}$ ) and supports both channel bonding and aggregation structures [9]. In channel aggregation, a discrete waveform forms each individual channel, while only waveform arranges at least two adjacent $2.16 \mathrm{GHz}$ channels. The mm-Wave signal in wireless communications carries out beam-forming technique in two directions; the first one is that two devices complete pre-coding where at the destination devices approximate correspondent channel then acknowledge the sender. The other is to transform analog beam on phased antenna arrays [10] in which spatial streams interference arise at the destination. In the IEEE 802.11ay protocol, a procedure for beam-forming technique for both SU-MIMO and MU-MIMO [11] communication systems are described. It allows communication devices to figure out the best transmitting and receiving configuration in concurrent transmission for multiple spatial streams. In next section, a brief scenario on both SU-MIMO and MU-MIMO features in this standard is explained. However, in wireless transmission a latency causing by propagating the signal to the receiver is unstable due to real-time based communication channels traffic. This also refers to a transmission delay of the packet while it is in wave. Most simulators allow scientists to investigate only constant latency in the experiment which is not supporting unexpected delays. This requires link capacity estimation for the accurate examination of PHY level efficiency. In this article, an implementation including unstable link capacity on PHY and MAC signal processing blocks upon IEEE 802.11ay standard is proposed.

\section{MILLIMETER WAVE SU/MU-MIMO COMMUNICATIONS}

The mm-Wave spectrum band in wireless communication depends on phased antenna arrays to attend the energy from targeted end upon analog beams development. Attaining digital beam-forming involves a committed radio frequency in mm-Wave for each element in antenna arrays. A hybrid beamforming [12] supports both digital and analog wave then a single mm-Wave is used for antenna elements, but solely one of those arrays is in progress at a time. Hybrid system is practically low power required while maintaining a performance compared to complete digital beam-forming. The pre-coding outlines spatial streams to all mm-Waves. To implement a MIMO communication, the source will retrieve a transmissionacknowledge (TACK). At this point, an EDMG device holds PHY carrier and disconnects. MIMO channels are lazy during spatial inter-frame stream until disconnection time turns zero as all source antennas tune into the TACK defined by a beam-forming procedure. In this situation, the device senses a TACK for a singleuser (SU-MIMO) communication. In downlink multi-users (MU-MIMO) communication, the access point (AP) is now acting as the source and requires the TACK instead.

\subsection{SU-MIMO beam-forming}

In this operation, a single stream is encoded, mixed up, and then projected to various spatial streams. The source then utilizes an autonomous modulation and coding scheme (MCS) [13] for individual stream. If spatial block coding time is active then each individual stream is designed to two spatial streams at different space-time. In that situation, a transferred stream over wireless communication does not relate to a MAC frame but corresponds to a fraction of frame as such. In the end, spatial streams are projected to multiple transmissions. To simulate SU-MIMO in this case, we require the preceding wave synchronization at the source and related part per se at the destination. The runtime of IEEE 802.11ay PHY layer simulation will rise as it relies on factors regarding the total number of packets, number of devices, and packet sizes. We expect this to be even worse when simulating the IEEE 802.11ay PHY layer. The reason is that the standard initiates billions of packets in a second. In orthogonal frequency division multiplexing (OFDM) modulation, multiple spatial streams transmission influences symbols in the frame's payload. This technique only needs a mapping table for SNR-to-PER. The table includes all SNRs of spatial streams, corresponds to number of sources and receivers, as well as channel complexity in each pair of antennas. 


\subsection{MU-MIMO beam-forming}

The AP transfers spatial streams to distinct receivers simultaneously in the MU-MIMO operation. Many scientists have been introducing separate scheduling algorithms [14] for MU-MIMO communications. These algorithms minimize interferences and SNR in the MU-MIMO communication to escalate available link capacity [15]. To run IEEE 802.11 ay MU-MIMO simulation, we propose two parameters regarding link capacity and network complexity. For the first parameter, the AP generates many spatial streams to individual device, and each device selects two or more mm-Wave to communicate. Here, this employment reflects an upper-bound on the channel's link capacity. It is equivalent to simulate the SU-MIMO as we eliminate inter-stream interference among the identical device's streams. We assume that analog beams are not orthogonal to eliminate device interferences. The impact of digital beam-forming simulation is focused. The overhead caused by the amount of devices in the MU-MIMO communication is also considered. In the second parameter, we implement each device in the network to have a single mm-Wave linked to an antenna to send/receive an individual stream. The AP has several mm-Wave chains, but it communicates a single stream per device. In this case, each stream accommodates a MAC packet for a particular device. Furthermore, we allow analog beam-forming to issue OFDM beams to avoid inter-stream interferences and as the result we can neglect digital beam-forming. Multibeam designed for communications using analog antenna arrays is discussed in [16].

\subsection{Additive white Gaussian noise (AWGN)}

A discrete AWGN [17] channel is regarded as the input-output variable function.

$$
a_{d}=b_{d}+n_{d}
$$

Where $n_{d}$ is symmetric complex Gaussian noise samples with variance $\sigma_{d}^{2}$. Given that in the memoryless system, the communication channel with AWGN can be defined as a pair of input/output variables as shown in Figure 1.

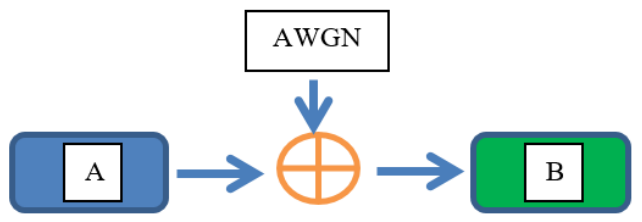

Figure 1. AWGN input/output model by (2).

For general key index $d$, the probability distribution function of AWGN channel can be represented

$$
p(b \mid a)=\frac{1}{\pi \sigma_{d}^{2}} \exp \left(-\frac{|b-a|^{2}}{\sigma_{d}^{2}}\right)
$$

In practical condition, the input variable which represents information stream $\mathrm{A}$ is encoded by a set of $\mathrm{K}$, where $K=\left\{A_{1}, A_{2}, \ldots, A_{K}\right\}$ with uniform probability $1 / K$. K-ary, phase shift keying (K-PSK), and $\mathrm{K}$-ary, quadrature amplitude modulation (K-QAM) are widely used modulations. For K-ary modulation with uniform probability, the mutual information $(M I)$ can be denoted as shown in (3) and approximated as appeared in (4), respectively.

$$
\begin{aligned}
& M I(A ; B)=\log _{2} K+\frac{1}{K} \sum_{n=1}^{K} \int p\left(b \mid A_{n}\right) \log _{2} \frac{p\left(b \mid A_{n}\right)}{\sum_{m=1}^{K} p\left(b \mid A_{m}\right)} d b \\
& M I(A ; B) \approx \log _{2} K+\frac{1}{M} \sum_{n=1}^{M} \log _{2} \frac{p\left(b_{n} \mid a_{n}\right)}{\sum_{m=1}^{K} p\left(b_{n} \mid A_{m}\right)}
\end{aligned}
$$

For the capacity of AWGN computation where a mean power constraint $\mathrm{E}\left\{|A|^{2}\right\}=H$, a symmetric complex Gaussian $(S)$ distribution with $\mathrm{SNR}, \mathrm{H} / \sigma_{d}^{2}$ can be written as (8).

$$
S=\log _{2}\left(1+\frac{\mathrm{H}}{\sigma_{d}^{2}}\right)
$$


The expression (5) can be calculated from input/output variables as well.

$$
S=\log _{2} \frac{\sigma_{b}^{2} \sigma_{a}^{2}}{\sigma_{a}^{2} \sigma_{b}^{2}-\left|\sigma_{a b}\right|^{2}}=\log _{2} \frac{1}{1-\left|\alpha_{a b}\right|^{2}}
$$

Where $\sigma_{b}^{2}$ and $\sigma_{a}^{2}$ are output and input variance accordingly. The $\sigma_{a b}$ is called the input/output covariance, and $\alpha_{a b}\left(=\sigma_{a b} /\left(\sigma_{b} \sigma_{a}\right)\right.$ represents the input/output correlation coefficient.

\section{PROPOSED MODEL AND EXPERIMENTAL ANALYSIS}

The performance evaluation of sender's mm-Wave is to quantify how perfect the Wireless LAN source devices are built. These devices include filtering, digital signal processing, amplification, and updown-link conversation. In addition, source also resolves the output mm-Wave quality using an error vector magnitude (EVM) [18]. It also encompasses the accuracy of channel center frequency and a voltage standing wave ratio (VSWR). Similarly, at the destination we quantify how well the receiver functions are. They are for instance amplification, up-down-link conversation, digital signal processing and signal filtering. Besides, inter-stream interference, clear channel assessment (CCA), the hold time for down-conversation, and the accuracy of received signal strength indication (RSSI) are apparently measured. IEEE 802.11ay which is referred to directional multi-gigabits (DMG) can offer digital throughputs up to $300 \mathrm{~Gb} / \mathrm{s}$ with short delay, utilizing the $60 \mathrm{GHz}$ band to achieve low inter-stream interference. Wi-Fi based on certified WiGig is introduced to fasten the availability speed of wireless devices, whose PHY/MAC design supports with this standard. WiGig will surge wireless network performance, ensuing improved network bandwidth and resembling channels. The 802.11 ay PHY aligns three modulated signals: i) Control PHY (beam-forming with BPSK modulation) compliments control with error detection and correction, ii) Single carrier PHY provides BPSK, QPSK, 16 QAM, and 64 QAM modulations with different rates of low density parity check (LDPC) $1 / 2,5 / 8,3 / 4,13 / 16$, and $7 / 8$, and iii) orthogonal frequency division multiplex PHY is based on multicarrier modulations to support higher throughput and modulation densities than the single carrier PHY. Note that the OFDM [19] operation mode is not commercially implemented yet. Modulation and coding scheme (MCS) of the SC PHY is shown in Table 1.

Table 1. MCS index for a SC PHY in IEEE 802.11ay

\begin{tabular}{|c|c|c|c|c|}
\hline Index & Enhanced DMG & Modulation & Coded bits per symbol in OFDM & LDPC \\
\hline MCS 1 & & $\pi / 2$-BPSK & 1 & $1 / 2$ \\
\hline MCS 2 & & $\pi / 2$-BPSK & 1 & $1 / 2$ \\
\hline MCS 3 & & $\pi / 2$-BPSK & 1 & $5 / 8$ \\
\hline MCS 4 & & $\pi / 2$-BPSK & 1 & $3 / 4$ \\
\hline MCS 5 & & $\pi / 2$-BPSK & 1 & $13 / 16$ \\
\hline MCS 6 & & $\pi / 2$-QPSK & 2 & $1 / 2$ \\
\hline MCS 7 & & $\pi / 2$-QPSK & 2 & $5 / 8$ \\
\hline MCS 8 & & $\pi / 2$-QPSK & 2 & $3 / 4$ \\
\hline MCS 9 & \multirow{5}{*}{ MCS 9.1} & $\pi / 2$-QPSK & 2 & $13 / 16$ \\
\hline & & $\pi / 2$-QPSK & 2 & $7 / 8$ \\
\hline MCS 10 & & $\pi / 2-16-\mathrm{QAM}$ & 4 & $1 / 2$ \\
\hline MCS 11 & & $\pi / 2-16-\mathrm{QAM}$ & 4 & $5 / 8$ \\
\hline MCS 12 & & $\pi / 2-16-\mathrm{QAM}$ & 4 & $3 / 4$ \\
\hline & MCS 12.1 & $\pi / 2-16-\mathrm{QAM}$ & 4 & $13 / 16$ \\
\hline & MCS 12.2 & $\pi / 2-16-\mathrm{QAM}$ & 4 & $7 / 8$ \\
\hline & MCS 12.3 & $\pi / 2-64-\mathrm{QAM}$ & 6 & $5 / 8$ \\
\hline & MCS 12.4 & $\pi / 2-64-\mathrm{QAM}$ & 6 & $3 / 4$ \\
\hline & MCS 12.5 & $\pi / 2-64-\mathrm{QAM}$ & 6 & $13 / 16$ \\
\hline & $\operatorname{MCS} 12.6$ & $\pi / 2-64-\mathrm{QAM}$ & 6 & $7 / 8$ \\
\hline
\end{tabular}

The block diagram for our proposed simulation model to investigate variable link latency in SU/MU-MIMO with mm-Wave for this standard [20] is presented in Figure 2. We will impose both AWGN and our link delay variable to the IEEE 802.11ay operation. The signal processing at the destination is digested as depicted in Figure 3.

\section{1. mm-Wave on 802.11ay network with link delay estimation}

In the subsequent section, we scrutinize the performance of MPFC [21] with unpredictable link delay in order to achieve the emergence in the developing IEEE 802.11ay wireless network. The experimental mm-Wave and Wi-Fi network is configured as described in Figure 4. The wireless network 
presented in Figure 4 where the multi-baseband-client at destination possesses an IEEE 802.11ay radio. The multi-baseband access point (MBAP) [22] can communicate through $60 \mathrm{GHz}$ links. The MBAP and clients are observing the MPFC. The proper routing configuration has been implemented to route all packets perfectly over wireless communication links. The offset mode [23] allows the 802.11ay link to be in a reserve condition for MPFC packets in a normal state. The IEEE 802.11ay link will be re-active for MPFC transmission as the back-off time in the standby mode is reached. In the experiment, we preserve the back-off mode (inactive and reactive period) by switching over the radio system. It is comparable to a jam period. During the investigation, we collect the PER values and compute the SNR per stream in each modulation and coding schemes as listed in Table 1, respectively.

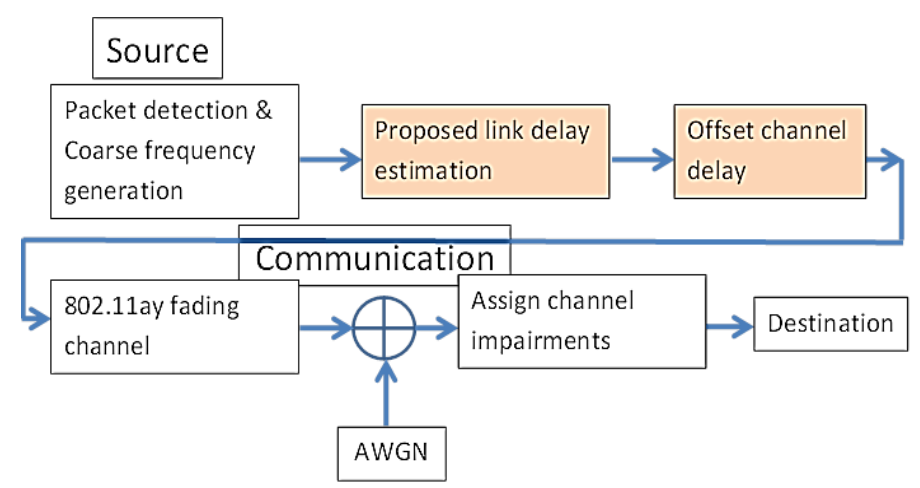

Figure 2. 802.11ay simulation blocks

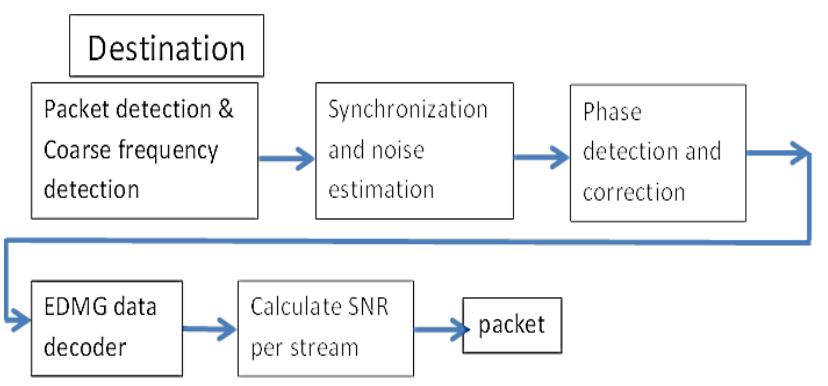

Figure 3. Signal operation blocks at the destination

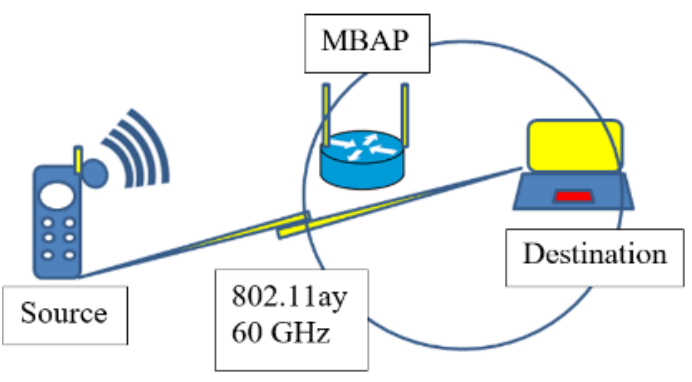

Figure 4. Wireless network for end-to-end simulation model

\subsection{Simulation results}

The IEEE 802.11ay guideline has endorsed the requirements of bandwidth intensive applications [24] in their transmissions in which the communication technology can be functional. We can summarize Scilab/Xcos simulator [25] environment into the following eight conditions as explained in Table 2. We thus investigate the impact of network congestion through randomized and unstable link delay on the performance metrics of SC PHY. We design to explore the low (30\% congestion) and high (80\% congestion) congested conditions on wireless 802.11ay link. We group and visualize the PER metrics at SNR attention (listed in Table 1 in Figures 5(a) and 5(b). In the figure, we also point out the average throughput of each MCS index as depicted in Figures 5(c) and 5(d), respectively. It is significantly seen that the PER values are higher in all indices. That means the MPFC has been affected due to the offset time operation from link delay. However, the employment of such packet size $(4.096 \mathrm{~KB})$ for this experiment should take into trade-off consideration. In addition, the open-space-wireless devices of end-to-end link have been used in the EDMG full-data-rate mode. We also can observe that the throughput metrics of PHY on 802.11ay are almost identical. It is because we tolerate the maximum PER is up to 10-2 at the SNR point. However, the higher congested mode has dropped a performance compared to the other slight congested one. The worst value of the throughput estimates the throughput of higher MCS index. The reason is that the unstable characteristics of two wireless links delay. The offset time confuses the packet scheduler of MPFC at maximum transmission rate, accordingly, lowering the whole throughput. 
Table 2. Parameters for simulator

\begin{tabular}{cc}
\hline Input Parameters & Descriptions \\
\hline Data bandwidth & At least $60 \mathrm{GHz}$. \\
Congested Link latency & Adaptive. \\
MPFC & EDMG with maximum data rate. \\
Mobility & {$[-20,+20]$ PPM $[26]$.} \\
Wireless environment & Open space hotspot. \\
Packet error rate (PER) @ SNR attention & $10^{-2}$. \\
Packet size & $4.096 \mathrm{~KB}$. \\
SC PHY & As appeared in Table 1. \\
\hline
\end{tabular}

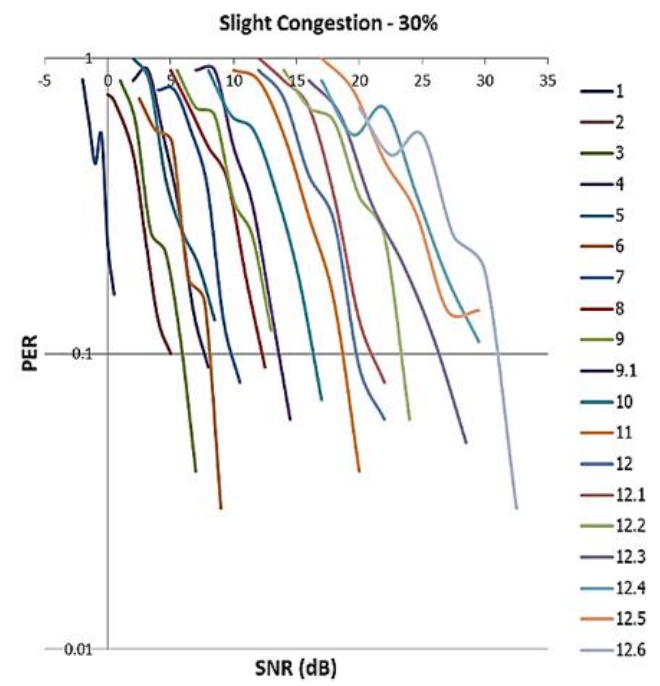

(a)

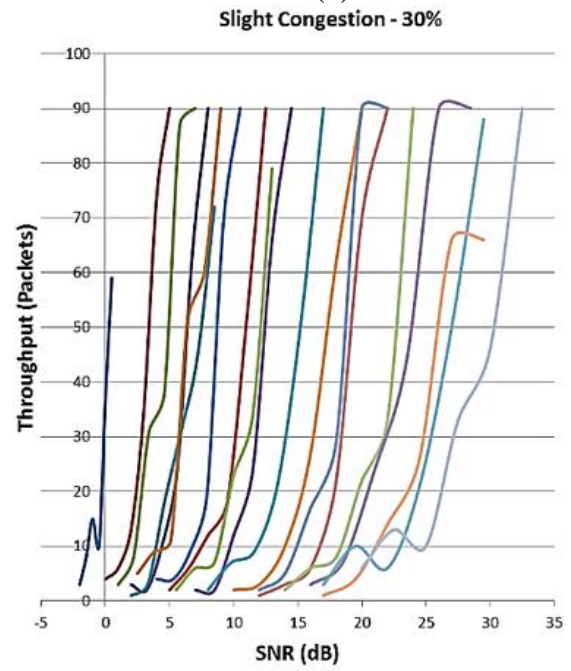

(c)

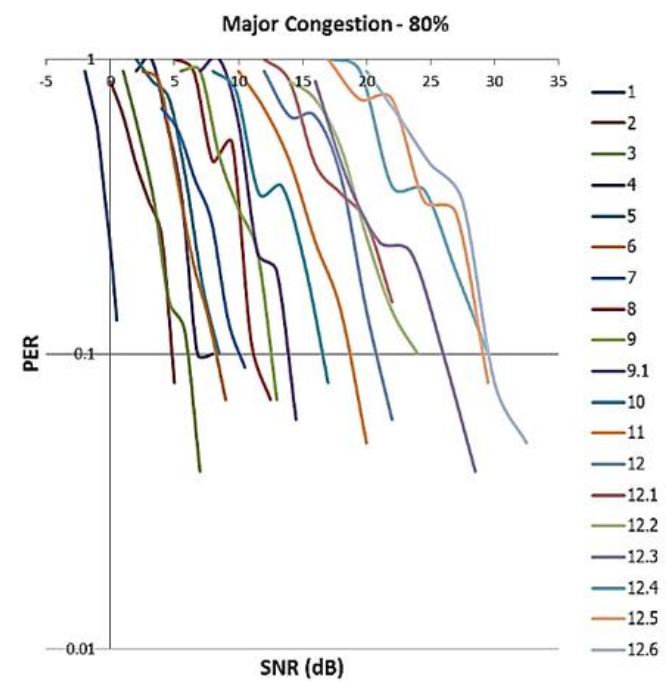

(b)

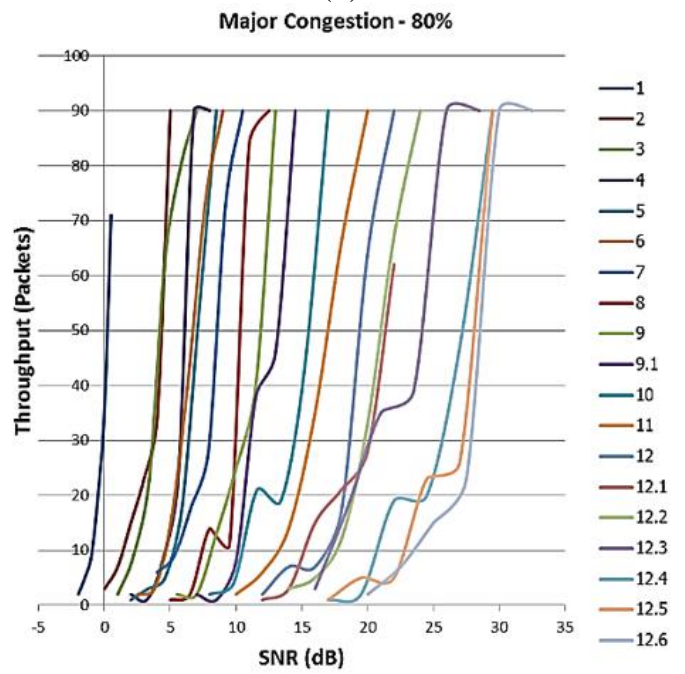

(d)

Figure 5. Simulation result: (a) PER at SNR attention-30\% congestion, (b) PER at SNR attention-80\% congestion, (c) throughput-30\% congestion, and (d) throughput- $80 \%$ congestion

\section{CONCLUSION}

With the outgrowth of the state-of-the-art IEEE 802.11ay wireless communication, which can support Gbps data rate on Wi-Fi connections, it is essential to examine the performance of IEEE 802.11ay SC PHY efficiency. This study exploits an end-to-end experimental bed on 802.11ay adaptive link delay in an open space environment under congested network and interference situations. We agree the multi-Gb/s throughput of both light and heavy traffic with the constraints of PER. We also affirm that the link delay problem (e.g., due to handshake, antenna directions or blockage) still arises. To iron out the problem, we further investigate the packet size effect as a medium which allows a rapid switch-over between SC PHY to a 
traditional Wi-Fi connection. Additionally, MPFC with the adaptive data rate control mode may adjust the traffic on IEEE 802.11ay to Wi-Fi link.

\section{REFERENCES}

[1] "Cisco annual internet report (2018-2023) white paper." https://www.cisco.com/c/en/us/solutions/collateral/executive-perspectives/annual-internet-report/white-paper-c11-741490.html (Accessed Jul. 2020).

[2] T. Norp, "5G requirements and key performance indicators," Journal of ICT, vol. 6, no. 1\&2, pp. 15-30, 2018, doi: $10.13052 /$ jicts $2245-800 X .612$

[3] M. Shafi et al., "5G: a tutorial overview of standards, trials, challenges, deployment, and practice," IEEE Journal on Selected Areas in Communications, vol. 35, no. 6, pp. 1201-1221, 2017, doi: 10.1109/JSAC.2017.2692307.

[4] T. Nitsche, C. Cordeiro, A. B. Flores, E. W. Knightly, E. Perahia, and J. C. Widmer, "IEEE 802.11ad: directional 60 GHz communication for multi-gigabit-per-second Wi-Fi," IEEE Communications Magazine, vol. 52, no. 12, pp. 132-141, 2014, doi: 10.1109/MCOM.2014.6979964.

[5] H. A. Leftah and H. N. Alminshid, "Channel capacity and performance evaluation of precoded MIMO-OFDM system with largesize constellation," International Journal of Electrical and Computer Engineering (IJECE), vol. 9, no. 6, pp. 5024-5030, 2019, doi: 10.11591/ijece.v9i6.pp5024-5030.

[6] S. Ismail, D. M. Ali, and A. L. Yusof, "MECC scheduling algorithm in vehicular environment for uplink transmission in LTE networks," International Journal of Electrical and Computer Engineering (IJECE), vol. 9, no. 2, pp. 1191-1200, 2019, doi: 10.11591/ijece.v9i2.pp1191-1200.

[7] H. Assasa, J. Widmer, J. Wang, T. Ropitault, and N. Golmie, “An implementation proposal for IEEE 802.11ay SU/MU-MIMO communication in ns-3," Proceedings of the 2019 Workshop on Next-Generation Wireless with, no. 3, 2019, pp. 26-29.

[8] Y. Ghasempour, C. R. C. M. da Silva, C. Cordeiro, and E. W. Knightly, "IEEE 802.11ay: next-generation 60 GHz communication for 100 Gb/s Wi-Fi," IEEE Communications Magazine, vol. 55, no. 12, pp. 186-192, 2017, doi: 10.1109/MCOM.2017.1700393.

[9] N. Khalil and A. Najid, "Performance analysis of 802.11ac with frame aggregation using NS3," International Journal of Electrical and Computer Engineering (IJECE), vol. 10, no. 5, pp. 5368-5376, 2020, doi: 10.11591/ijece.v10i5.pp5368-5376.

[10] T. C. Quyen, "Phased antenna arrays toward 5G," IntechOpen, pp. 1-11, 2020, doi: 10.5772/intechopen.93058.

[11] B. Archana and T. P. Surekha, "Integrated approach for efficient power consumption and resource allocation in MIMOOFDMA," International Journal of Electrical and Computer Engineering (IJECE), vol. 10, no. 2, pp. 2069-2076, 2020, doi: 10.11591/ijece.v10i2.pp2069-2076.

[12] F. Sohrabi and W. Yu, "Hybrid digital and analog beamforming design for large-scale antenna arrays, ” IEEE Journal of Selected Topics in Signal Processing, vol. 10, no. 3, pp. 501-513, 2016, doi: 10.1109/JSTSP.2016.2520912.

[13] H. Hamlili, S. Kameche, and A. Abdelmalek, "S $\alpha$ S noise suppression for OFDM wireless communication in rayleight channel," International Journal of Electrical and Computer Engineering (IJECE), vol. 10, no. 2, pp. 2003-2010, 2020, doi: 10.11591/ijece.v10i2.pp2003-2010.

[14] M. K. Husein, "Minimize MIMO OFDM interference and noise ratio using polynomial-time algorithm," International Journal of Electrical and Computer Engineering (IJECE), vol. 10, no. 1, pp. 900-907, 2020, doi: 10.11591/ijece.v10i1.pp900-907.

[15] R. Pal, K. V. Srinivas, and A. K. Chaitanya, "a beam selection algorithm for millimeter-wave multi-user MIMO systems," IEEE Communications Letters, vol. 22, no. 4, pp. 852-855, 2018, doi: 10.1109/LCOMM.2018.2803805.

[16] J. A. M. Zhang et al., "Multibeam for joint communication and radar sensing using steerable analog antenna arrays," IEEE Transactions on Vehicular Technology, vol. 68, no. 1, pp. 671-685, 2019, doi: 10.1109/TVT.2018.2883796.

[17] P. N. Alevizos and A. Bletsas, "Sensitive and nonlinear far-field RF energy harvesting in wireless communications," IEEE Transactions on Wireless Communications, vol. 17, no. 6, pp. 3670-3685, 2018, doi: 10.1109/TWC.2018.2812889.

[18] J. Wang, Q. Wu, D. Wang, C. Zhang, and W. Wu, "Reducing error vector magnitude of OFDM signals using threshold vector circle method," Proceedings of Fourth International Conference on Wireless and Optical Communications, 2016, doi: $10.1117 / 12.2262936$.

[19] D. Z. Blanco, C. A. A. Meza, S. M. Sánchez, and A. D. Firoozabadi, "OFDM tolerance to additive white Gaussian and laser phase noises in optical heterodyning systems accompanied by the feasible pilot-assisted equalization," Optical Review, vol. 27, pp. 156-169, 2020, doi: 10.1007/s10043-020-00587-8.

[20] P. Zhou et al., "IEEE 802.11ay-based mmWave WLANs: design challenges and solutions," IEEE Communications Surveys and Tutorials, vol. 20, no. 3, pp. 1654-1681, 2018, doi: 10.1109/COMST.2018.2816920.

[21] J. Liu, "Wireless multipath fading channels modeling and simulation based on sum-of-sinusoids," IEEE International Conference on Computer Communication and the Internet, 2016, pp. 165-168, doi: 10.1109/CCI.2016.7778900.

[22] F. A. P. De Figueiredo et al., "A baseband wireless spectrum hypervisor for multiplexing concurrent OFDM signals," Sensors, vol. 20 , no. 4 , pp. 1-34, 2020, doi: 10.3390/s20041101.

[23] K. Nguyen et al., "Performance evaluation of IEEE 802.11ad in evolving Wi-Fi networks," Wireless Communications and Mobile Computing, vol. 2019, 2019, pp. 1-11, Art. no. 4089365, doi: 10.1155/2019/4089365.

[24] Y. Li and D. Liu, "An efficient bandwidth reservation policy for data-intensive applications in cloud," International Journal of Networking and Virtual Organisations, vol. 21, no. 4, pp. 438-454, 2019.

[25] V. Singh, H. G. Navada, and K. N. Shubhanga, "Large power system stability analysis using a FOSS-based tool: SciLab/Xcos," in Proceedings of the National Power Systems Conference, 2018, pp. 1-6, doi: 10.1109/NPSC.2018.8771782.

[26] G. Scarano, A. Petroni, M. Biagi, and R. Cusani, "Blind fractionally spaced channel equalization for shallow water PPM digital communications links,” Sensors, vol. 19, no. 21, 2019, Art. no. 4604, doi: 10.3390/s19214604 